\title{
Fe-based Soft Magnetic Materials for Electronic Devices
}

\author{
Denisa Olekšáková ${ }^{1, *}$ \\ 1 Department of Applied Mathematics and Informatics, Faculty of Mechanical Engineering, Technical University in Košice, Letná 9, 04200 Košice, Slo- \\ vak Republic
}

\begin{abstract}
The amorphous and crystalline ferromagnetic alloys are known as materials with excellent soft magnetic properties. These alloys have been intensive studied during last decades due to their mechanical and magnetic properties and they are challenge for scientists to extend research of these materials with the aim to broaden their technical applications. Fe and Ni-based alloys exhibit very good soft magnetic properties with near-to-zero magnetostriction. This property renders this material as a potential candidate for a differently of industrial applications (for example electronic security sensors, magnetic recording heads and others). The aim of this work is to find out the best soft magnetic material for the preparing components and pieces for modern electronic devices.
\end{abstract}

Keywords: soft magnetic materials, coercivity, magnetization, powder, mechanical milling, mechanical alloying.

\section{Introduction}

Modern electronic devices (such as digital telecommunication equipments, railway or automotive techniques, power supplies and others) supply of inductive components or magnetic cores with compact volumes and high universality in geometric shape and magnetic properties [1].

To realize such a power core materials are needed that exhibit an excellent combinations of soft magnetic properties as: near-to-zero magnetostriction, low core loss, low coercivity and high permeability and saturation induction. For performance applications but also to achieve good frequency properties of coercivity and permeability and eddy current losses must be as low as possible [2]. For large scale industrial production the material must to exhibit the reliability to produce and to be easy to work of cores to electronic components. Moreover, the raw material should be easy to obtain and no expensive. The polycrystalline material FeNi is very suitable (excellent soft magnetic properties, cheap material...) and very well known in low energy cores of transformers, anchors of electromotors and cores of magnetic recording heads. The shape, in which are usually these materials prepared (conventional sheets, crystalline ribbon prepared by rapid solidification technology...), is in many cases not suitable shape for industry applications. One of the ways to prepare bulk material (suitable for industrial applications) is the hot compaction of powder, which can be produced by the mechanical milling of thin ribbon, mixture of powders, sheets...

The mechanical ball milling technique has been successfully used to prepare many alloys in powder form, which are suitable for hot or cold compaction into a 
variety of shapes [3]. Mechanical milling is a nonequilibrium process for materials synthesis. This dry and high or low energy milling process has attracted considerable interest in recent years. This proces was applied to a wide range of materials, leading to unstable and metastable phases under highly non-equilibrium conditions [4].

\section{Experimental material and methods parameters}

The aim of this work was to prepare powder samples of permalloy ( $\mathrm{Fe}_{19} \mathrm{Ni}_{81}$, wt. \%) by mechanical milling of microcrystalline ribbon $\mathrm{Fe}_{19} \mathrm{Ni}_{81}$ (sample I) and that of pure elements Fe and $\mathrm{Ni}$ (sample II) in a vibratory mill in range from 0 to 1000 hours and to investigate their magnetic properties with the sense to find the best material for the preparing bulk samples.

The sample I was prepared by mechanical milling of a microcrystalline $\mathrm{Fe}_{19} \mathrm{Ni} \mathrm{Ni}_{1}$ (wt. \%) ribbon, prepared by melt spinning method, in a ball vibratory mill for 1000 h. Sample II was prepared by mechanical alloying of pure elements Fe and $\mathrm{Ni}$ (Fe: Ni = 19:81, wt. \%) in a ball vibratory mill, too. The milling was performed under argon atmosphere and this process has been interrupted at various times of mechanical milling to remove a part of milled sample (powder) for investigations of magnetic properties.

Thermomagnetic curves were measured by vibratory sample magnetometer in magnetic field of $0.3 \mathrm{~T}$ with heating rate of $10^{\circ} \mathrm{C} / \mathrm{min}$. The coercivity and magnetic moment of all powder samples were measured by vibratory sample magnetometer as a function of the milling time in magnetic field with maximum induction of $0.6 \mathrm{~T}$ at room temperature.

The crystalline character of all powder samples before and after mechanical milling/alloying was confirmed by X-ray diffraction.

\section{Results and discussion}

Fig. 1 displays thermomagnetic curves of sample I for different times of the mechanical milling. The magnetization decreases to zero at $550^{\circ} \mathrm{C}$ and it corresponds with the Curie temperature of the conventional Fe19Ni81 alloy (conventional sheets) [5]. The behavior of all thermomagnetic curves is monotonous without inflection points and we have supposed that system is a single- phase during all time of mechanical milling. This leads to the result that the mechanical milling of the sample I consists mainly of crushing the parts of crystalline ribbon to smaller and smaller particles (gradually) and the producing of fine powder with the increasing of the milling time. The Curie temperature was calculated from these thermomagnetic curves (fig. 1).

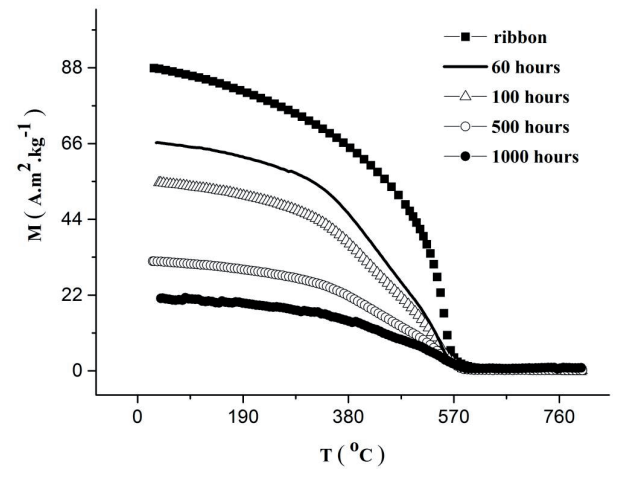

Fig. 1: The thermomagnetic curves of sample / for different periods of the mechanical milling.

The behavior of the coercivity and the magnetization for sample I depending on the milling time is noted in Fig. 2. We can see that magnetic moment monotonously decreases during all the milling time. It can be caused by the increasing of the volume fraction of fine particles exhibiting such size, in which the domain structure is not formed and external magnetic field (0.6 T) is not enough for the producing of the saturation state by the rotation of the magnetization vector. The next reason may be the formation of such small particles, which exhibit superparamagnetic behavior with blocking temperature in above mentioned external field below room temperature.

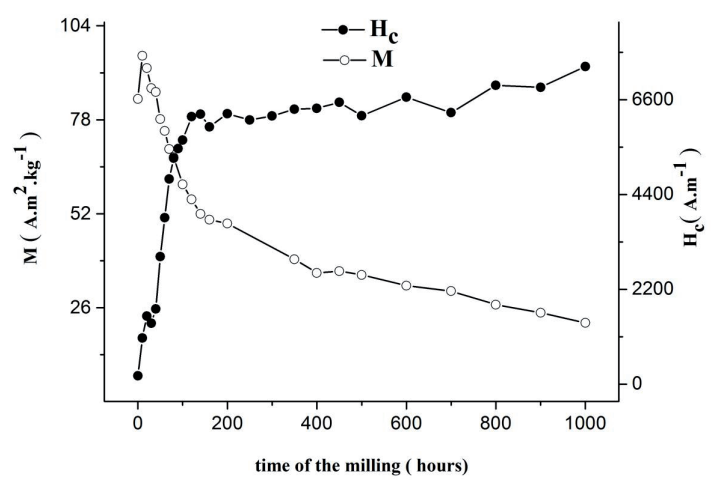

Fig. 2: The coercivity and the magnetization of sample I. 
At the beginning of the mechanical milling the coercivity increases up to approximately 6300 A.m -1 $^{-1}$ for 140 hour of the milling. It can be caused by a gradual reduction of particles size (Fig. 2). Magnetization process of the sample $I$ is less and less realized by the domain wall displacement and dominant process becomes the rotation of magnetization vector. A large amount of the sample I consists of single-domain particles and magnetization process is realized by the magnetization rotation only in these particles.

Thermomagnetic curves of sample II are noted in Fig. 3. At the beginning of the mechanical milling (to 30 hours) each thermomagnetic curve is a superposition of two independent thermomagnetic curves (pure iron and pure nickel). After 30 hours of milling, we can see the formation of new phases with different Curie temperature. New created phases were identified by their Curie temperature, which was obtained from thermomagnetic curves (fig. 3). We detected following phases:

- pure nickel with $T_{C}=360^{\circ} \mathrm{C}$,

- pure iron with $T_{c}=765^{\circ} \mathrm{C}$,

- Fe ${ }_{40} \mathrm{Ni}$ i6o phase with $\mathrm{Tc}_{\mathrm{c}}=623^{\circ} \mathrm{C}$ (samples milled from 30 to 200 hours),

- Fe0.5Nig5.5 phase with $T_{C}=434^{\circ} \mathrm{C}$ (samples milled more than 200 hours).

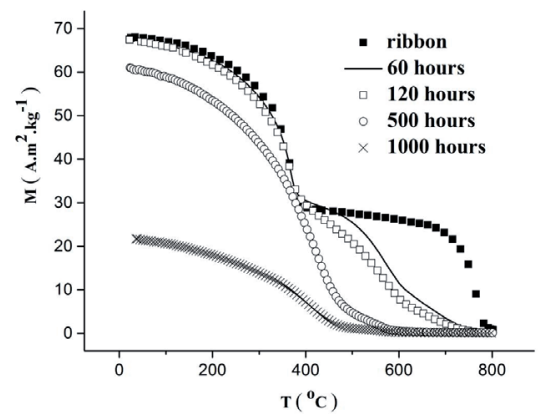

Fig. 3: The thermomagnetic curves of sample II for different periods of the mechanical milling.

The behavior of the coercivity and the magnetization of the sample $\|$ is displayed in fig. 4 and this is similar and caused by the similar mechanisms as in sample I. The small deviation from the monotonous behavior is caused by the formation and disappearance of various phases with different magnetization during all mechanical alloying.

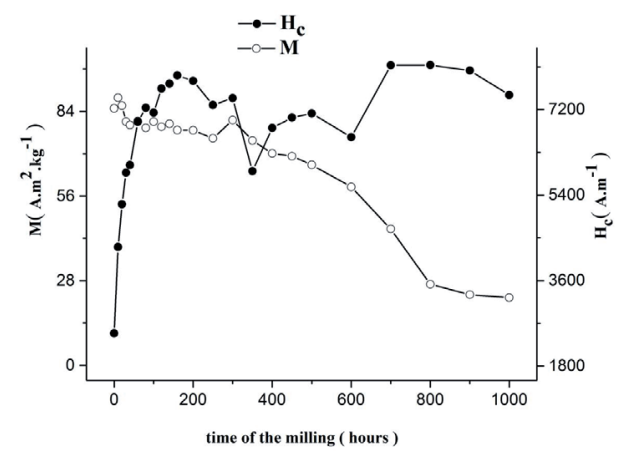

Fig. 4: The coercivity and the magnetization of sample II.

\section{Conclusions}

By the investigating the influence of the mechanical milling and the mechanical alloying in a vibratory ball mill on the magnetic properties of powder FeNi alloys we have found that:

1. Sample I prepared by the mechanical milling of the crystalline ribbon $\mathrm{Fe}_{19} \mathrm{Ni}_{81}$ (wt. \%) is a solid solution with stable structure during all time of the mechanical milling.

2. Sample II prepared by mechanical alloying of Fe and Ni powder mixture (the same chemical composition like sample I) consists of various phases exhibiting various Curie temperatures which are formed and disappear during all mechanical alloying.

3. Sample I is more suitable powder material for prepare bulk samples (for example by the hot compaction of powder prepared by the mechanical milling) in the various shape for industrial applications.

\section{Acknowledgments \\ This work was supported by the projects APVV-15-0115 and KEGA 072TUKE-4/2014.}

\section{References and Notes}

[1] G. Herzer, Acta Materialia 61 (2013) 718-734.

[2] J. Petzold, Scripta Materialia 48 (2003) 895-901.

[3] J. Füzer, P. Kollár, D. Olekšáková, S. Roth, Journal of Alloys and Compounds 483 (2009) 557-559.

[4] C. Suryanarayana, E. Ivanov, V. V. Boldyrev, Mater. Sci. Eng. A, 304-306 (2001) 151-158.

[5] T. F. Connolly, E. D. Copenhaver, Bibliography of magnetic matarials and tabulation of magnetic transition temperature, 1970.

[6] D. Olekšáková, J. Füzer, P. Kollár, T. Švec, J. kováč, J. Briančin, K. polański, Czechoslovak Journal of Physics 54 (2004) D93-D96. 
Acta Mechanica Slovaca

Journal published by Faculty of Mechanical Engineering - Technical University of Košice

\section{Biographical notes}

RNDr. Denisa Olekšáková, PhD., (born in 1979) received PhD degree in Physics of Condensed Matter and Acoustics at the P. J. Šafárik University in Košice in 2007. At present, she works as a lecturer at the Department of Applied Mathematics and Informatics of the Faculty of Mechanical Engineering of Technical University in Košice. Her research interest is the investigation of the structure and magnetic properties of soft magnetic materials. She is co-author of 50 scientific publications and 16 of these publications are registered in the Current Contents database, for which are more than 50 citations were recorded in the citation databases SCI and SCOPUS. 